\title{
Low Complexity Code Design for the 2-user Gaussian Multiple Access Channel
}

\author{
Aline Roumy \\ IRISA-INRIA \\ 35042 Rennes cedex, France \\ aroumy@irisa.fr
}

\author{
David Declercq \\ ETIS ENSEA-UCP CNRS UMR 8051 \\ 95014 Cergy cedex, France \\ declercq@ensea.fr
}

\author{
Eric Fabre \\ IRISA-INRIA \\ 35042 Rennes cedex, France \\ fabre@irisa.fr
}

We address the problem of designing good codes for the Gaussian multiple access channel (MAC). The corner points of the capacity region have long been known to be achievable by single user decoding. This idea was also used to achieve any point of the capacity region by means of rate splitting [4]. Here we focus on the design of multiuser codes since the key idea for achieving any point in the capacity region of the Gaussian MAC is random coding and optimal joint decoding. We rather consider a suboptimal but practical approach that consists in using irregular Low-Density Parity-Check codes (LDPC) decoded with Belief Propagation (BP) [1, 2]. As in [1], we tackle the difficult and important problem where all users have the same power constraint and the same rate in order to show that the designed multiuser codes can get close to any point of the boundary in the capacity region of the Gaussian MAC.

Hence, 2 independent users $x^{(1)}$ and $x^{(2)}$ are sent to a single receiver. Each user is LDPC encoded by different irregular LDPC codes with codeword length $N$. The codeword is BPSK modulated and the synchronous discrete model of the transmission at time $n$ is given by, $\forall 0 \leq n \leq N-1$

$$
y_{n}=x_{n}^{(1)}+x_{n}^{(2)}+w_{n}=\left[\begin{array}{ll}
1 & 1
\end{array}\right] \cdot Z_{n}+w_{n}
$$

where $Z_{n}=\left[x_{n}^{(1)}, x_{n}^{(2)}\right]^{T}$ is the state vector of the multiuser channel, and $w_{n}$ is a zero mean additive white Gaussian noise with variance $\sigma^{2}$. This 2-user LDPC-MAC system is best described by its factor graph composed of the 2 LDPC graphs, which are connected through function nodes representing the link between the state vector $Z_{n}$ and the coded symbols of each user $x_{n}^{(1)}$ and $x_{n}^{(2)}$. In order to analyze this multiuser MAC decoder, we formulate density evolution (DE) [3] and study the stability condition of the fixed point corresponding to zero BER.

Proposition 1. The local stability condition of the DE for the 2-user Gaussian MAC is the same as that of the single user case.

The optimization criterion is to maximize $R$ subject to a vanishing Bit Error Rate, where this constraint is expressed in terms of DE. However, DE is computationally intensive and in order to reduce the computational burden of LDPC optimization, faster techniques have been proposed, based on the approximations of DE by a one-dimensional dynamical system $[6,5]$, and reference therein. Several recent works show that an accurate single parameter is the mutual information between the variables associated with the variable nodes and their messages (see $[6,5]$ ). The mutual information evolution describes each computation node in BP-decoding by a mutual information transfer function, which is usually referred to as the EXtrinsic mutual Information Transfer (EXIT) function. For codes with binary variables only, the EXIT charts can be expressed analytically [5], leading to very fast and powerful optimization algorithms.
For the state check node update (the state variable is no more binary), we express analytically the EXIT chart based on two different approximations. We assume that the distribution of the messages at the state check node input is either Gaussian (Gaussian Approximation: GA) or the output of a binary erasure channel (Erasure channel approach: EC). It follows that for the MAC multiuser decoder:

Proposition 2. The stability condition for the approximated DE (GA) is the same as that of the exact DE whereas the stability condition for $(E C)$ is less stringent than that of the exact DE.

As in the single user case, we wish to have a low-complexity optimization algorithm and propose new hypotheses:

$\left\{\mathcal{H}_{0}\right\}$ : hypothesis equal LDPC codes. The 2 LDPC codes belong to the same ensemble.

$\left\{\mathcal{H}_{1}\right\}$ : hypothesis without interleaver. Each and every state check node is connected to two variable nodes (one in each LDPC code) having exactly the same degree.

Proposition 3. Under hypotheses $\mathcal{H}_{0}$ and $\mathcal{H}_{1}$, the evolution of the mutual information is linear in the parameters of the codes to be optimized. The optimization can thus be solved using linear programming.

\begin{tabular}{|c|c|c|c|c|}
\hline Rate & 0.3 & 0.4 & 0.5 & 0.6 \\
\hline \hline Gap to Shannon limit (GA) & 0.22 & 0.15 & 0.19 & 0.52 \\
\hline Gap to Shannon limit (EC) & 0.38 & 0.26 & 0.21 & 0.59 \\
\hline
\end{tabular}

Codes are optimized with approximated methods but evaluated with full DE. As expected, the GA approach gives slightly better codes than the EC approach. Moreover, since the best LDPC codes designed with full DE [1] only slightly outperform our designed codes $(0.19 \mathrm{~dB}$ instead of $0.18 \mathrm{~dB})$, our design methods emerge as attractive choices.

\section{REFERENCES}

[1] A. Amraoui, S. Dusad, R. Urbanke, "Achieving general points in the 2-user Gaussian MAC without time-sharing or rate-splitting by means of iterative decoding", in (ISIT 2002), July 2002.

[2] A. DeBaynast, D. Declercq, "Gallager Codes for Multiple Access", in (ISIT 2002), July 2002.

[3] T.J. Richardson, M.A. Shokrollahi, R.L. Urbanke, "Design of capacity-approaching irregular low-density parity-check codes," IEEE Trans. on Inf. Theory, pp. 619-637, Feb. 2001.

[4] B. Rimoldi and R. Urbanke, "A rate splitting approach to the Gaussian multiple-access channel," IEEE Trans. on Inform. Theory, vol. 42, no. 2, pp. 364-375, March 1996.

[5] A. Roumy, S. Guemghar, G. Caire, S. Verdú, "Design methods for irregular repeat accumulate codes," IEEE Trans. on IT.

[6] S. ten Brink, "Designing iterative decoding schemes with the extrinsic information transfer chart," $A E \ddot{U}$ Int. J. Electronic. Commun., vol. 54, no. 6, pp. 389-398, Dec. 2000. 